\title{
Wie sich Kollagenosen auf der Haut verraten
}

\author{
Kollagenosen sind Systemerkrankungen, die jedes Organ betreffen können. Dies erklärt das \\ "bunte“ klinische Bild, das die Diagnosestellung oft erheblich erschwert. Da in vielen Fällen \\ Hautveränderungen zu den wichtigsten Manifestationen dieser Erkrankungen gehören, \\ sollten Sie dem Organ Haut besondere Beachtung schenken.
}

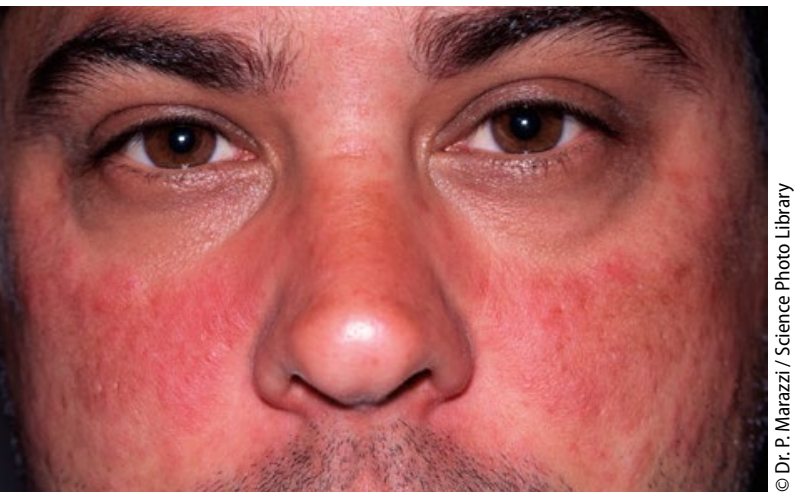

Schmetterlingserythem bei Lupus erythematodes.

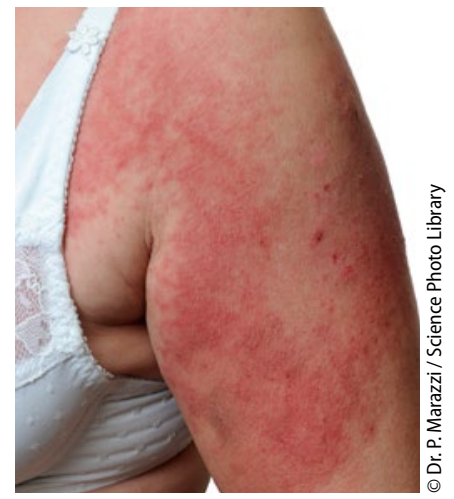

Ausschlag bei Dermatomyositis.

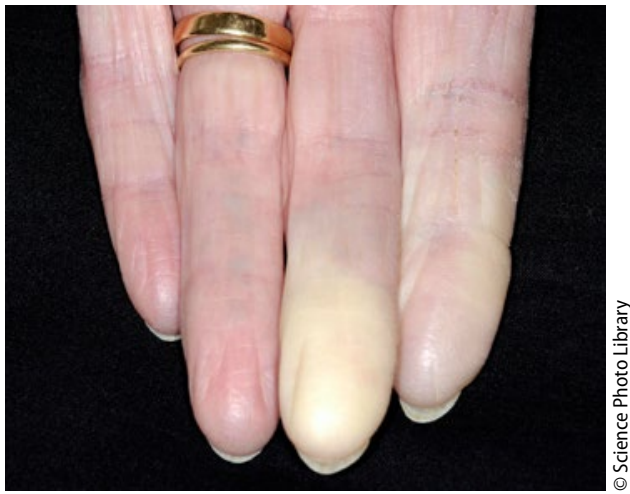

Raynaud-Phänomen am Finger.
_ Traditionsgemäß werden zu den Kollagenosen der Lupus erythematodes (LE), die Dermatomyositis und die systemische Sklerose, früher als Sklerodermie bezeichnet, gerechnet, obwohl diese Krankheitsbilder unterschiedliche pathogenetische Mechanismen aufweisen. erläuterte Prof. Michael Hertl, Marburg.

\section{Erhöhte Photosensitivität beim Lupus erythematodes}

Typisch für den LE ist eine gestörte UVToleranz, die zu einer Apoptose epidermaler Keratinozyten führt. Dies ist der Auslöser für die Bildung von Autoantikörpern, die gegen verschiedene Komponenten des Organismus gerichtet sind und Immunkomplexe bilden, die wiederum zu einer Vaskulitis im Bereich der Nieren- oder Hautgefäße führen können.

Man unterscheidet die primär kutane und die systemische Form des LE. Typischerweise kommt es bei der kutanen Form zu einem Exanthem an den lichtexponierten Hautarealen, bei dessen Abheilung sogar Vernarbungen auftreten können.
Typisch für den systemischen LE sind persistierende, aber auch flüchtige Exantheme, eine erhöhte Lichtempfindlichkeit, chronisch-atrophische Plaques oder eine Livedo-Vaskulopathie. Dazu kommen eine Serositis, z. B. ein Pleuraerguss oder ein Aszites, eine Beteiligung von Niere bzw. ZNS und hämatologische und immunologische Veränderungen wie Anämie, Leukopenie und/oder Thrombopenie.

\section{Bei Dermatomyositis auf}

\section{Tumorsuche gehen}

Die Dermatomyositis kann in jedem Lebensalter auftreten. Das Häufigkeitsmaximum ist das 50. Lebensjahr, Frauen sind häufiger als Männer betroffen. „Bei Erwachsenen Dermatomyositis-Patienten sollte immer an eine Paraneoplasie gedacht und eine entsprechende Tumorsuche veranlasst werden“, so Hertl. Typisch sind heliotrope Exantheme mit sekundärer Poikilodermie in Form von Atrophien, Teleangiektasien und Hypopigmentierungen an lichtexponierten Hautarealen. Dazu kommen Papeln an den Grund- und Mittelgelenken der
Hände und Nagelfalzhyperkeratosen mit Teleangiektasien und Splitterblutungen. Die Muskelbeteiligung führt zu einer proximal betonten symmetrischen Muskelschwäche. Die Muskelenzyme sind erhöht. Gesichert wird die Diagnose durch eine Biopsie.

\section{Raynaud-Syndrom bei systemischer Sklerose}

Die systemische Sklerose befällt ebenfalls neben der Haut auch innere Organe sowie das Skelettsystem. Typisch ist die kutane Sklerose, die diffus oder akral betont auftritt. Initial klagen Betroffene häufig über ein Raynaud-Syndrom und Teleangiektasien im Gesichtsbereich. Betroffen ist nicht selten auch der Ösophagus in Form einer Motilitätsstörung, die sich als Dysphagie manifestiert.

Weitere Komplikationen sind die Lungenfibrose, die Nephrosklerose, die pulmonale Hypertonie und die Myokardfibrose.

\section{Dr. Peter Stiefelhagen}

- 25. Fortbildungswoche für praktische Dermatologie und Venerologie, 29.7.2016 in München 